\title{
Le vivier de Katoro (Istrie, Croatie)
}

\section{Marie-Brigitte Carre et Tea Katunarić}

Musée d'Umag, Centre Camille Jullian (Université Aix-Marseille / CNRS) et Centre Ausonius (Université de Bordeaux 3 / CNRS) (éd.)

\section{(2) OpenEdition Journals}

\section{Édition électronique}

URL : http://journals.openedition.org/cefr/233

DOI : $10.4000 /$ cefr.233

ISSN : 2282-5703

Éditeur

École française de Rome

Référence électronique

Marie-Brigitte Carre et Tea Katunarić, «Le vivier de Katoro (Istrie, Croatie) », Chronique des activités archéologiques de l'École française de Rome [En ligne], Balkans, mis en ligne le 19 décembre 2012, consulté le 12 septembre 2019. URL : http://journals.openedition.org/cefr/233; DOI : 10.4000/ cefr.233

Ce document a été généré automatiquement le 12 septembre 2019.

(c) École française de Rome 


\section{Le vivier de Katoro (Istrie, Croatie)}

\section{Marie-Brigitte Carre et Tea Katunarić}

Musée d'Umag, Centre Camille Jullian (Université Aix-Marseille / CNRS) et Centre Ausonius (Université de Bordeaux 3 / CNRS) (éd.)

1 Le vivier de Katoro, qui appartient à un vaste complexe comprenant des structures portuaires autour d'une grande villa, est signalé depuis la seconde moitié du XIX ${ }^{e}$ siècle ${ }^{1}$. Une fouille de sauvetage réalisée par N. Bolšec Ferri sur la partie nord de la villa en 2003 et 2006 permit la découverte de plusieurs pièces avec des mosaïques, de thermes et d'une double sépulture aristocratique ${ }^{2}$. L'embarcadère a été fouillé en 2001-2002 ${ }^{3}$. En revanche, le vivier, bien connu par un relevé remarquable exécuté en 1929 par l'ingénieur Cusmani sous l'égide d'A. Degrassi, qui l'avait repéré lors de ses promenades archéologiques le long de la côte istrienne, n'avait jamais fait l'objet d'une étude détaillée ${ }^{4}$. Le savant décrivait alors, sur la rive sud du promontoire de Catoro / Katoro, une grande construction en arc de cercle, appuyée aux rochers du rivage sur une longueur de $70 \mathrm{~m}$. La superficie en était divisée en deux parties par un gros mur rectiligne, long de $55 \mathrm{~m}$ environ, tandis que d'autres murs limitaient de plus petits espaces de moins de $30-35 \mathrm{~m}^{2}$ (fig. 1). 
Fig. 1 - Katoro. Photo aérienne du vivier.

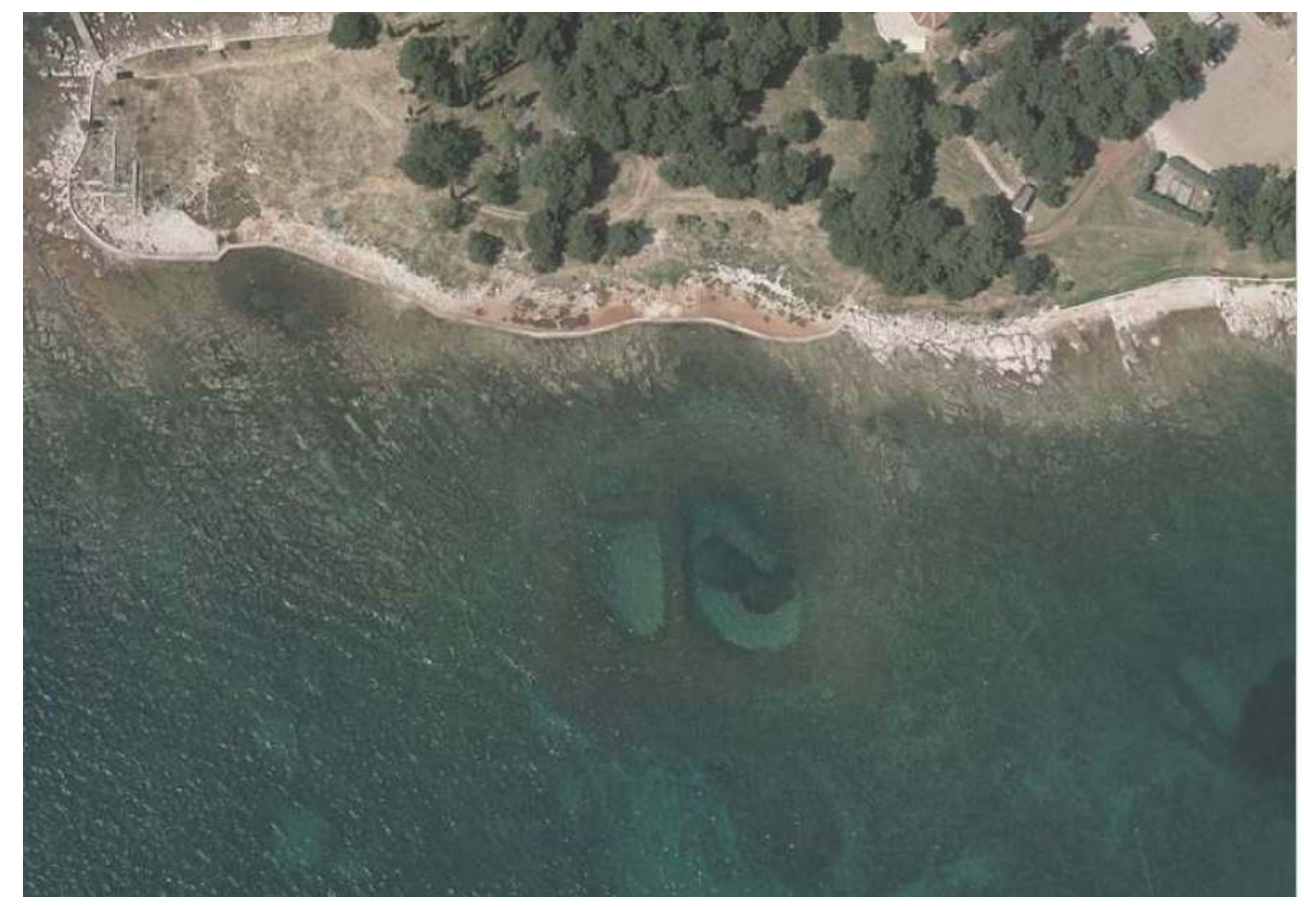

Institut géodésique de Rijeka.

Contre l'opinion de ses prédécesseurs qui y voyaient un ou deux ports, Degrassi interpréta cette structure comme un vivier à poissons, une piscina appartenant à la villa.

Ce vivier se rattache à une typologie spécifique de la côte istrienne à l'époque romaine ${ }^{5}$. Ces grandes structures (leur surface interne est comprise entre 1500 et $2200 \mathrm{~m}^{2}$ ) composées de deux à quatre bassins sont en effet caractérisées par le mode de construction de leurs larges murs périmétraux dont la fondation est faite de moellons jetés en vrac depuis la surface. Les fouilles récentes de trois viviers à S. Bartolomeo / Jernejeva draga et Fizine ${ }^{6}$ en Slovénie, et à Kupanja ${ }^{7}$ en Croatie, ont renouvelé l'intérêt pour celui de Katoro, plus anciennement connu, et dont le plan apparemment plus complexe pouvait permettre de répondre aux nombreuses questions qui restent posées sur le fonctionnement et l'utilisation des ces aménagements (infra). En outre, on attendait de son étude des éléments de réponses aux discussions en cours sur le niveau de la mer antique sur la côte d'Istrie depuis les hypothèses émises par E. Fouache dès $2001^{8}$.

4 Une brève campagne de relevés eut lieu en mai $2009^{9}$. L'objectif en était d'évaluer les moyens nécessaires à la fouille de cette grande structure submergée. Depuis 2003, les recherches subaquatiques sur le littoral du Parentin, financées principalement par le Ministère français des Affaires étrangères et européennes, sont intégrées dans le programme L'Istrie et la mer dirigé par Francis Tassaux ${ }^{10}$, et sont conduites dans le cadre d'un partenariat entre le Zavičajni muzej Poreštine (Poreč), le Centre Camille Jullian (UMR 7299, Université d'Aix-Marseille-CNRS) et le Centre Ausonius-Université de Bordeaux 3. L'intervention sur le vivier de Katoro, qui dépend du Musée de Umag, a été rendue possible suite à un accord signé en 2008 entre cette institution dirigée par Biljana Bojiće, le Centre Ausonius et l'École française de Rome. Ont participé à cette enquête F. Tassaux (Bordeaux 3-Centre Ausonius); L. Damelet, photographe et V. Dumas, 
topographe (Centre Camille Jullian); G. Chapelin, architecte, S. Venet, ingénieur de recherche, Communauté d'agglomérations du Douaisis; B. Raffone, chargé de cours (Université d'Urbino); L. Claquin, L. Delavault et A. Perrier (étudiants en Master 2 à l'Université de Provence).

Les structures les plus apparentes ont fait l'objet d'un nettoyage superficiel mais l'essentiel du travail a consisté en une série de relevés topographiques mis en œuvre par Vincent Dumas (CCJ) : un Modèle Numérique de Terrain (MNT), pour lequel 1300 points ont été pris ; une planimétrie de l'ensemble de la structure ; et enfin trois coupes relevées le long de lignes matérialisées sur le fond. Ces lignes ont servi de guide pour un piquetage dans le sable qui a permis de préciser la profondeur du fond des bassins et avoir une idée de la conformation de ce même fond dans l'objectif d'une future fouille.

Les premières observations confirment l'exactitude du plan et des observations d'A. Degrassi. Le vivier, situé au pied d'un escarpement rocheux, s'ancre sur le platier qui limite la côte (fig. 2 et 3).

Fig. 2 - Katoro. Plan.

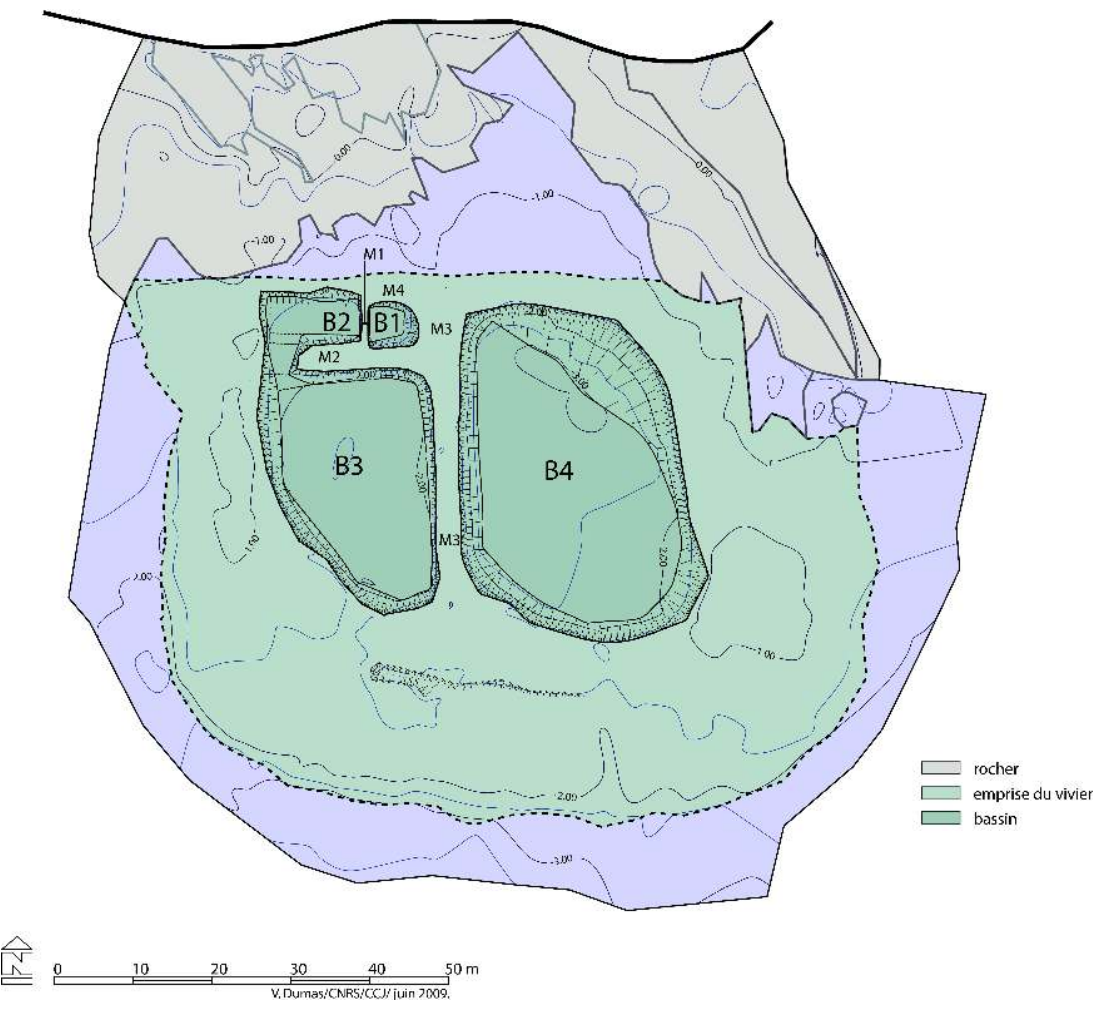

V. Dumas, CNRS-CCJ. 
Fig. 3 - Katoro. Vue axonométrique.

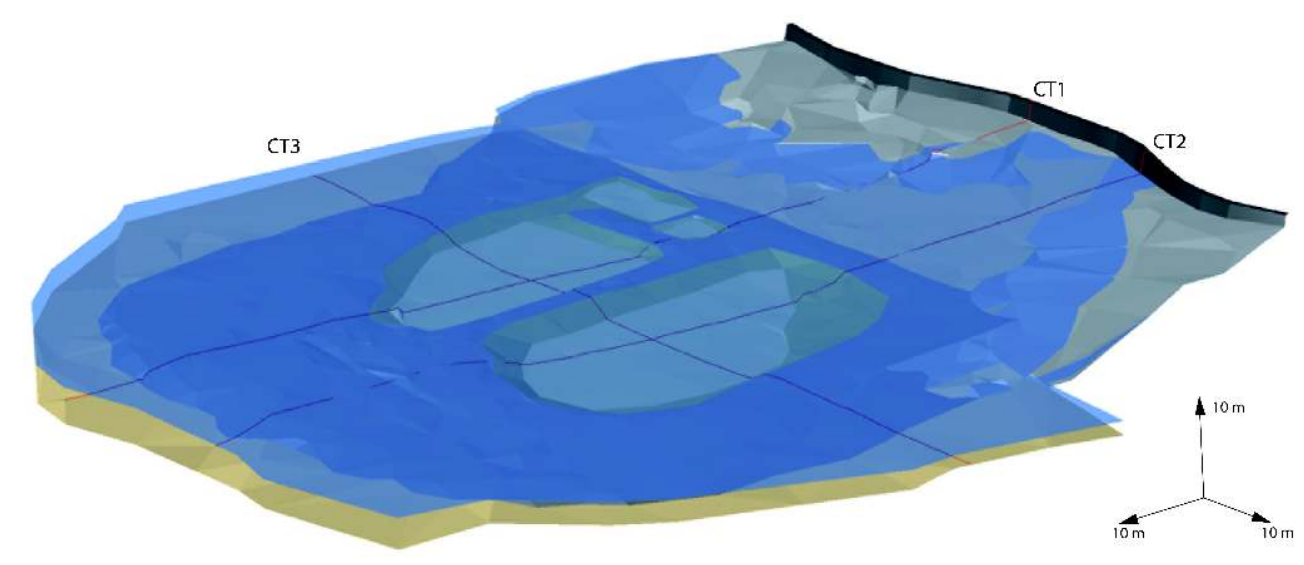

V. Dumas, CNRS-CCJ.

7 Il se présente sous la forme de quatre bassins ceints de murs plus ou moins bien conservés. Les vestiges occupent une surface totale d'environ $5465 \mathrm{~m}^{2}$, pour une longueur de $97,55 \mathrm{~m}$ du nord au sud et une largeur maximum de 70,32 $\mathrm{m}$ d'est en ouest. Ces dimensions, fondées sur une première estimation de la dispersion des blocs de l'enceinte extérieure, devront cependant être précisées. La profondeur atteint $-3,26 \mathrm{~m}$ tandis que les points les plus hauts ont été repérés à $-0,58 \mathrm{~m}$ sous le niveau moyen de la mer.

8 L'édifice est composé de quatre bassins de dimensions inégales (fig. 2). À l'est, le bassin B4 $\left(1083 / 671 \mathrm{~m}^{2}\right)^{11}$, dont l'enceinte extérieure est formée de blocs jetés en vrac, est divisé du bassin B3 $\left(560 / 416 \mathrm{~m}^{2}\right)$ par le mur M3. Ce dernier doit probablement être identifié avec le muraglione repéré par A. Degrassi, construit en gros blocs superposés régulièrement. Le fond, dont la surface a été repérée au moyen d'un piquetage dans le sable le long de deux axes nord-sud, est presque horizontal (fig. 4). La hauteur du sable, inférieure à un mètre dans B3, est supérieure dans le bassin 4 à la hauteur de la pique d'1,80 $\mathrm{m}$ utilisée pour les mesures. Le niveau du fond des bassins peut donc être estimé entre 4 et $5 \mathrm{~m}$ sous celui de la mer actuelle. 
Fig. 4 - Katoro. Coupes transversales.
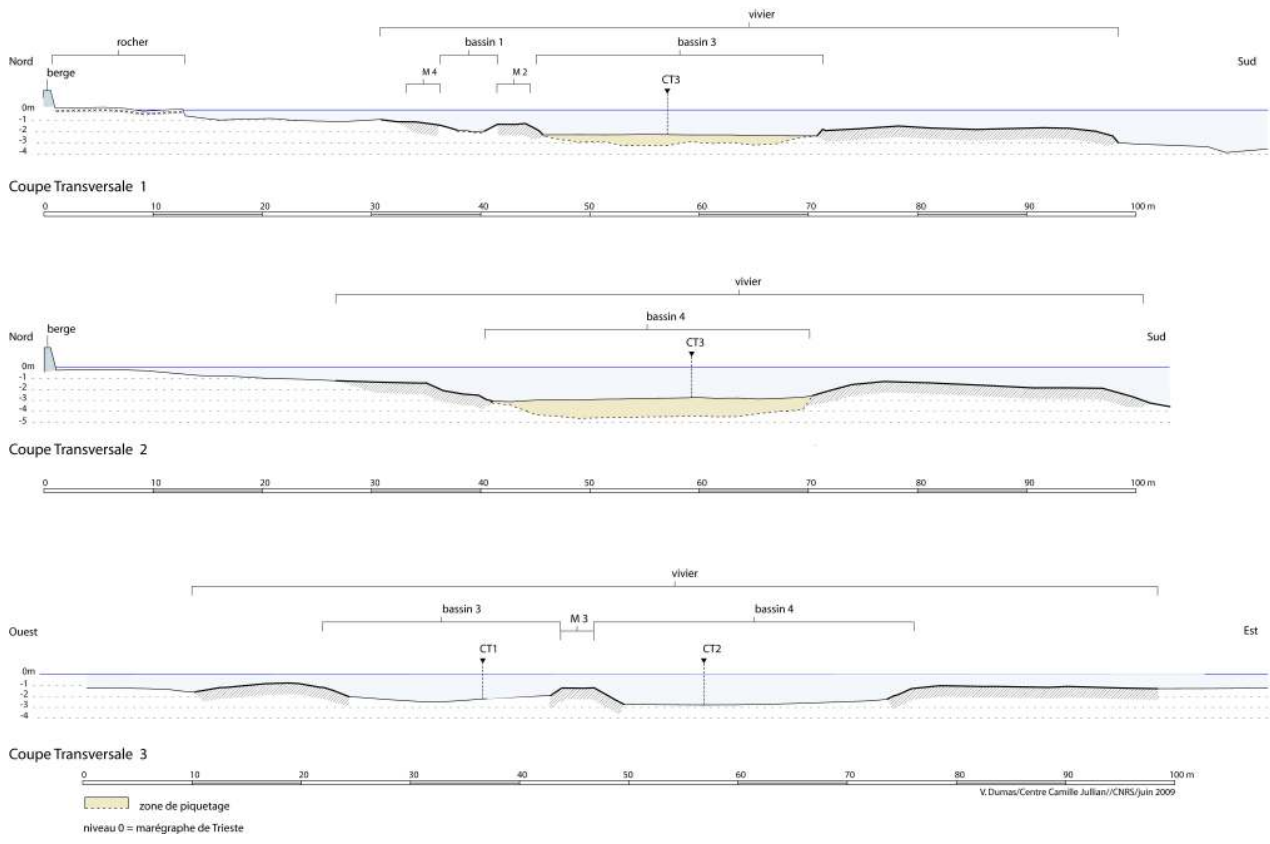

V. Dumas, CNRS-CCJ

9 La partie septentrionale di B3 est subdivisée à son tour en deux autres bassins. B1, le plus petit $\left(34 / 16 \mathrm{~m}^{2}\right)$, est complètement fermé. Les murs 1 et 2 , qui le délimitent à l'ouest et au sud, sont formés de petits moellons et, pour l'instant, aucune trace de mortier ou d'un autre liant n'a été observée. Le mur 1 , qui le sépare du bassin 2, a été partiellement nettoyé (fig. 5).

Fig. 5 - Katoro. L'assise supérieure du mur 1 entre les bassins 1 et 2.

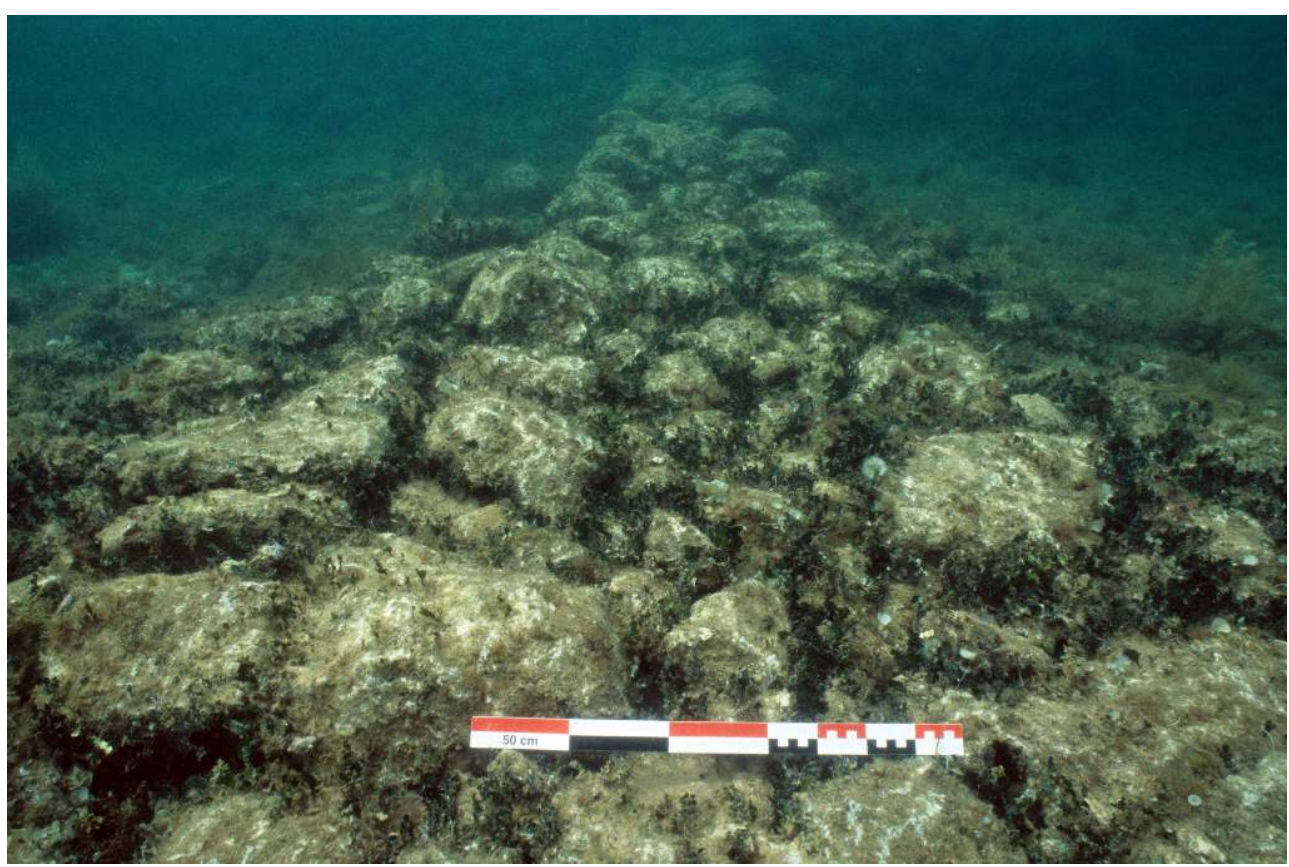

Cliché L. Damelet, CNRS-CCJ. 
assises sont conservées sur une hauteur de $0,60 \mathrm{~m}$. Un sondage pratiqué dans son angle sud-ouest a permis d'entrevoir la dernière assise posée directement sur le fond rocheux. Deux gros fragments de tegulae sont insérés dans l'effondrement du mur. Le mur 2 n'a pas été dégagé mais les deux alignements de ses parements sont parfaitement visibles.

Au nord, le mur M4 paraît appuyé contre un ressaut rocheux dans le platier, mais cette observation demandera un complément d'enquête. Bien que le parement ouest de M3 n'ait pas été vu sous l'effondrement du mur, son prolongement au sud, dans le bassin 3 témoigne qu'il devrait à son tour être construit comme les précédents. Atteint sous une mince couche de sable, le fond de ce bassin est à un peu plus de deux mètres sous le niveau actuel de la mer. sont éparpillés à proximité. Ils évoquent les alignements disposés sur le sommet des fondations en pierres jetées des autres viviers d'Istrie. À Fizine, la partie haute est constituée de deux rangées de blocs massifs entre lesquelles se trouve un blocage de pierres sans mortier ; à S. Bartolomeo, une série de grands blocs pouvant atteindre $3 \mathrm{~m}$ de long repose sur le radier ${ }^{12}$. Ici aussi, ces éléments devaient à la fois rehausser les murs externes au-dessus du niveau de la mer et protéger les installations des plus grandes vagues et du vent du sud (scirocco), qui entre dans la baie avec violence.

De nombreux tessons d'amphores et de vaisselle ont été repérés en surface, à l'intérieur ou à proximité de la structure. On ne peut pas pour l'instant déterminer s'il s'agit de mobilier lié à sa construction ou à son utilisation ou, plus probablement, de déblais consécutifs à l'abandon de la villa. Néanmoins, la découverte de fragments de tuiles dans le mur M1 confirme la datation de l'édifice à l'époque romaine.

Les grands viviers à fondations en pierres jetées de la côte istrienne présentent des caractéristiques profondément originales ${ }^{13}$. L'amplitude de la marée, exceptionnelle pour la Méditerranée, explique peut-être des aménagements spécifiques au golfe adriatique, qui impliquent un type de construction particulièrement massif. Ces bassins ne sont pas étanches et ont besoin d'être complètement immergés pour fonctionner (fig. 6) en permettant le renouvellement de l'eau de mer à travers les gros radiers de fondation. Pour l'instant, aucune des quatre piscinae mises au jour n'a livré de vestiges permettant d'envisager une adduction d'eau douce. 
Fig. 6 - La mise en eau du vivier selon différents scénarios de remontée du niveau marin.

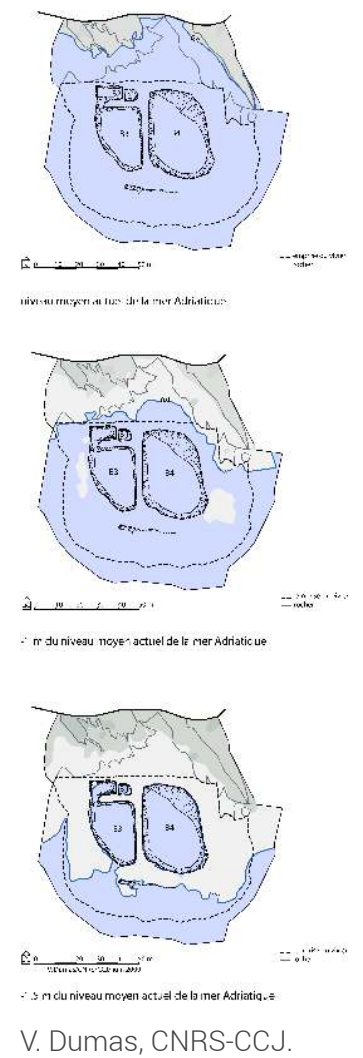

16 Avec une superficie utile de 1760/1160 $\mathrm{m}^{2}$ à l'intérieur des quatre bassins, ce vivier est le plus petit de la côte istrienne. La présence des murs de division, délimitant les petits bassins B1 et B2 qui scindent la partie septentrionale de B3, est un élément qui différencie la structure de Katoro des autres vivaria qui se présentent sous la forme de bassins accolés. L'utilisation de ces plus petits espaces, dont la profondeur lorsqu'ils étaient en fonction ne devait pas excéder $1 \mathrm{~m}$ si l'on admet une hypothèse d'un mètre de remontée du niveau de la mer depuis l'Antiquité ${ }^{14}$, n'est pas élucidée pour l'instant. Cependant, le principe de la séparation des tailles et des espèces est bien attesté dans les textes anciens et l'examen de l'ouverture du bassin B2 pourrait apporter des éléments supplémentaires d'information.

De même que le fonctionnement de ces grands viviers n'est pas encore connu, en l'absence de tout aménagement que l'on pourrait rapprocher de ce que préconisent les sources anciennes, aucune des hypothèses formulées sur leur exploitation n'est totalement satisfaisante : s'il s'agit de stockage de poisson pêché avant sa transformation, on ne trouve pas de comparaison pour des structures aussi vastes dans les aires géographiques où se trouvent les grandes usines de salaison; installations qui, au demeurant, manquent encore en Istrie. Pour sa part, l'hypothèse de l'élevage se heurte à la question du nombre de consommateurs potentiels dans une zone où l'on ne trouve pas de métropole comparable à Rome sur la côte tyrrhénienne, même si les ports de l'Istrie ( Parentium, Pola, Trieste) et ceux d'Aquilée, Ravenne ou Salone, peuvent avoir joué un rôle important dans la consommation de poisson frais. Au demeurant ces deux propositions ne s'excluent pas car elles pourraient avoir suivi un rythme saisonnier ${ }^{15}$. Malgré ces incertitudes, les textes nous apprennent que la rentabilité des viviers était assurée malgré 
des coûts de construction et de fonctionnement très élevés, ce qui correspond parfaitement à leur implantation dans les grands domaines istriens prévus pour assurer à leur propriétaire une rentabilité maximum. Comme sur la côte occidentale de l'Italie, les viviers sont toujours associés à des établissements côtiers. Une villa semble avoir été présente à proximité de celui de San Bartolomeo / Jernejeva draga, celui de Fizine s'insère dans un grand complexe portuaire ; le vivier de Kupanja s'intègre dans le complexe horsnorme de Loron, qui devient propriété impériale à la fin du $\mathrm{I}^{\mathrm{er}} \mathrm{s}$. de notre $\mathrm{n}$. è. ${ }^{16}$. Les dates de construction et les durées d'utilisation restant inconnues, leur exploitation peut s'inscrire aussi bien dans le développement de cette activité lucrative dans les domaines aristocratiques de la côte istrienne que dans le cadre d'une appropriation similaire à celle qui vit les piscinae tyrrhéniennes entrer dans le patrimoine impérial.

\section{BIBLIOGRAPHIE}

Bolšec 2006 = N. Bolšec, Katoro-Rt Tiola, dans Hrvatski Arheološki Godisnjak, 3, 2006, p. 235-237.

Carre-Auriemma 2009 = M.-B. Carre, R. Auriemma, Piscine e vivaria nell'Adriatico settentrionale : tipologie e funzioni, dans S. Pesavento Mattioli, M.-B. Carre (éd.), Olio e pesce in epoca romana : produzione e commercio nelle regioni dell'Alto Adriatico [Atti del Convegno, Padova, 16 febbraio 2007], Rome, 2009 (Antenor Quaderni, 14), p. 81-98.

Carre - Kovačić - Tassaux 2011 = M.-B. Carre, V. Kovačić, F. Tassaux, L'Istrie et la mer. La côte du Parentin dans l'Antiquité, Bordeaux, 2011 (Ausonius-Mémoires, 25).

Degrassi 1955 = A. Degrassi, I porti romani dell'Istria, dans Anthemon. Scritti di Archeologia e di Antichità Classiche in onore di Carlo Anti, Florence, 1955, p. 119-169.

Fouache et alii 2011 = É. Fouache, S. Faivre, J.-J. Dufaure, M. Ghilardi et alii, 5000 ans d'évolution relative du niveau marin en Istrie : qu'en est-il à l'époque romaine?, dans Carre - Kovačić - Tassaux 2011, p. 69-88.

Gluščević - Bolšec Ferri 2003 = S. Gluščević, N. Bolšec Ferri, Izvješće o podmorskim arheološkim radovima u Katoru kod Umaga, Obavijesti, br.1. god XXXV, dans HAD Zagreb, 2003, p. 114-120.

Katunarić 2009 = T. Katunarić, Katoro - vivarij, dans Hrvatski Arheološki Godišnjak, 6, p. 346-349.

Katunarić - Carre 2009 = T. Katunarić, M.-B. Carre, Sistematsko podvodno arheološko istraživanje rimskog ribnjaka, vivaria, u Katoru kod Umaga, dans Hrvatski arheološki godišnjak, 6, 2009, p. 346-349.

Matijašić 1998 = R. Matijašić, Gospodartsvo antičke Istre, Pula, 1998.

Stokin et alii 2008 = M. Stokin, A. Gaspari, S. Karinja, M. Erič, Archaeological Research of maritime infrastructure of Roman settlements on the Slovenian coast of Istria (1993-2007), dans R. Auriemma, S. Karinja (éd.), Terre di mare. L'archeologia dei paesaggi costieri e le variazioni climatiche [Atti del Convegno internazionale di studi, Trieste, 8-10 novembre 2007], Trieste-Piran, 2008, p. 56-74. Tassaux - Matijašić - Kovačić 2001 = F. Tassaux, R. Matijašić, V. Kovačić, Loron (Croatie), un grand centre de production d'amphores à huile istrienne (I Ir-IV $^{e}$ s. p.C.), Bordeaux, 2001 (Ausonius-Mémoires, 6). 


\section{NOTES}

1. Matijašić 1998, p. 127-128.

2. Bolšec 2006.

3. Gluščević - Bolšec Ferri 2003.

4. Degrassi 1955, p. 140-141 et pl. IV, 7.

5. Carre - Auriemma 2009.

6. Stokin et al. 2008.

7. Carre - Kovačić - Tassaux 2011, p. 161-166.

8. Fouache et alii 2011.

9. Katunarić - Carre 2009.

10. MEFRA 122-1, 2010, p. 228-235.

11. Les deux mesures correspondent respectivement à la surface totale et à la seule surface du fond du bassin. La surface utile se situe entre ces deux extrêmes, elle pourra être calculée plus précisément après la fouille.

12. Stokin et alii 2008, p. 60 et 71.

13. Cet aspect a été développé de façon exhaustive par R. Auriemma dans Carre - Auriemma 2009, p. 84-91. Une structure similaire est peut-être présente dans l'île de Svršata, archipel des Kornati / Incoronate.

14. Fouache et alii 2011.

15. Carre - Kovačić - Tassaux 2011, p. 167-172.

16. Tassaux - Matijašić - Kovačić 2001.

INDEX

Mots-clés : Istrie, Histria, vivier, villa maritime

Index géographique : Croatie, Katoro

institutions Musée d'Umag, Centre Camille Jullian (Université Aix-Marseille / CNRS), Centre Ausonius (Université de Bordeaux 3 / CNRS)

\section{AUTEURS}

\section{MARIE-BRIGITTE CARRE}

Centre Camille Jullian (Université Aix-Marseille / CNRS) - Carre[at]mmsh.univ-aix.fr

\section{TEA KATUNARIĆ}

Umjetnicka akademija Split (Croatie) - teakatunaric[at]gmail.com 